\title{
Expression of microRNAs that regulate bone turnover in the serum of postmenopausal women with low bone mass and vertebral fractures
}

\author{
Maria P Yavropoulou1, Athanasios D Anastasilakis², Polyzois Makras \\ Dimitrios G Tsalikakis ${ }^{4}$, Maria Grammatiki ${ }^{1}$ and John G Yovos ${ }^{1}$ \\ ${ }^{1} 1$ st Department of Internal Medicine, Laboratory of Clinical and Molecular Endocrinology, AHEPA \\ University Hospital, Aristotle University of Thessaloniki, Thessaloniki, Greece, ${ }^{2}$ Department of \\ Endocrinology, 424 General Military Hospital, Thessaloniki, Greece, ${ }^{3}$ Department of Endocrinology and \\ Diabetes, 251 Hellenic Air Force \& VA General Hospital, Athens, Greece, and ${ }^{4}$ Department of Informatics \\ and Telecommunication Engineering, University of Western Macedonia, Kozani, Greece
}

\author{
Correspondence \\ should be addressed \\ to M P Yavropoulou \\ Email \\ margia@med.auth.gr
}

\begin{abstract}
Background: Circulating microRNAs (miRs) are currently being investigated as novel biomarkers for osteoporosis and osteoporotic fractures.

Aim: The aim of this study was to investigate serum levels of specific microRNAs, known regulators of bone metabolism, in postmenopausal women with low bone mass and with or without vertebral fractures (VFs). Methods: For the analysis, 14 miRs were isolated from the serum of 35 postmenopausal women with low bone mass and with at least one moderate VF and 35 postmenopausal women with low bone mass without fractures. Thirty postmenopausal women with normal BMD values and no history of fractures served as controls. Main outcome parameters were changes in the expression of selected miRs in the serum of patient population and compared with controls.
\end{abstract}

Results: From the 14 miRs that were selected, we identified 5 miRs, namely miR-21-5p, miR-23a, miR-29a-3p, miR-124-3p and miR-2861 that were significantly deregulated in the serum of patients with low bone mass compared with controls. Serum miR-124 and miR-2861 were significantly higher, whereas miR-21, miR-23 and miR-29 were lower in patients compared with controls. In a sub-group analysis of the patient population, the expression of miR-21-5p was significantly lower among osteoporotic/osteopenic women with VFs, showing $66 \%$ sensitivity and $77 \%$ specificity in distinguishing women with a vertebral fracture.

Conclusion: This study identifies a differential expression pattern of miR-21-5p in the serum of women with low BMD and VFs.

\section{Introduction}

Osteoporosis is a systemic skeletal disorder characterized by low bone mass and impaired bone microarchitecture due to imbalance between bone formation and resorption during the remodeling cycle, leading to decreased bone strength and increased risk of fragility fractures $(1,2)$.

In current clinical practice, measurement of bone mineral density (BMD) by dual-X-ray absorptiometry
(DXA) (3) and calculation of fracture risk assessment (FRAX) score (4) are used for the evaluation of fracture risk. Despite the technological improvements of the last decades in the diagnostic equipment, with more advanced DXA machines and software, the estimation of fracture risk is still far from being optimal. BMD values measured by DXA demonstrate significant overlap between patients www.eje-online.org DOI: 10.1530/EJE-16-0583
두 2017 European Society of Endocrinology Printed in Great Britain
Published by Bioscientifica Ltd. 
with and without fragility fractures (5). Furthermore, the predictive value of FRAX score among patients with an available BMD measurement is likely to be suboptimal in patients with low lumbar spine but preserved femoral neck BMD (6).

In recent years, epigenetics presents as a new discipline that attempts to explain significant differences in phenotypes among patients with the same disease, such as diabetes mellitus or cancer (7). In contrast to the other epigenetic mechanisms that modulate gene transcription, micro RNAs (miRs), that are small non-coding RNAs, acting at the post-transcriptional level, directly modulate the gene expression of mRNA genes through the formation of an RNA-induced silencing complex, which leads to mRNA target cleavage and degradation and translation repression (8).

Several in vitro and ex vivo studies have reported the role of miRs in the regulation of cellular functions of bone cells such as proliferation, differentiation and apoptosis (9). Tissue expression of certain miRs is highly associated with their presence in the serum of osteoporotic patients (10). Furthermore, expression of certain miRs in the serum of patients with osteoporotic fractures may positively or negatively affect osteoblast activity (11), suggesting that circulating levels of these miRs may be directly linked to altered bone metabolism.

Given the important role of osteoblast (12), and osteoclast (13) function and survival in the development and progress of osteoporosis, circulating miRs that control their operational status could be used as biomarkers of decreased bone mass and increased fracture risk. To address this issue, we investigated the differential expression of specific miRs known to affect osteoblast and osteoclast function in osteoporotic and osteopenic women with or without vertebral fractures (VFs).

\section{Patients and methods}

\section{Study design}

This was a multicenter cross-sectional observational study that included 70 postmenopausal women with osteopenia/ osteoporosis in the lumbar spine (LS) and/or total hip (TH) or femoral neck (FN). Thirty-five of them had at least one moderate fragility VF, Grade 2 according to the Genant's semiquantitative method, whereas the remaining 35 had no history or presence of VFs in recent X-rays. Exclusion criteria were (i) history or presence of bone disease other than primary osteoporosis (e.g. primary or secondary hyperparathyroidism), (ii) medication known to affect bone metabolism (e.g. glucocorticoids, calcimimetics or anti-osteoporotic agents with the exception of previous treatment with denosumab and teriparatide with at least one-year drug-free period before enrollment), (iii) hip fracture or hip or knee replacement within 6 months before enrollment, (iv) history of high energy fractures, (v) any type of cancer, (vi) renal and/or liver failure, (vii) diabetes mellitus, (viii) uncontrolled hypothyroidism or hyperthyroidism and (ix) vitamin D deficiency (defined as serum $25-\mathrm{OH}$-vitamin D levels $<20 \mathrm{ng} / \mathrm{dL}$ ). Thirty healthy postmenopausal women with normal BMD and no history or X-ray evidence of fracture were recruited from the AHEPA hospital personnel and included in the study as controls. The quantification of bone mineral density $\left(\mathrm{BMD}, \mathrm{g} / \mathrm{cm}^{2}\right.$ ) was performed using dual-energy X-ray absorptiometry (DXA) at the dominant proximal FN and TH and the LS (L2-L4). All participants had standardized radiographs in antero-posterior and left lateral projections of the thoracic and lumbar spine and were asked about a history of fracture at non-vertebral sites. The radiographs were examined by an experienced skeletal radiologist. Body mass index (BMI) was calculated for each participant as the ratio between weight $(\mathrm{kg})$ and height squared $\left(\mathrm{m}^{2}\right)$. The study was approved by the Institutional Review Board of AHEPA University Hospital (Approval Number: 2468), and all participants gave their informed consent.

\section{Patient's characteristics}

Approximately 90\% ( $n=62)$ of the patients were treatment naïve at the time of the sampling, whereas 8 patients had previously received anti-osteoporotic treatment as following: teriparatide $20 \mu \mathrm{g} /$ day $(n=5)$ and denosumab $60 \mathrm{mg} / 6$ months $(n=3)$. Drug-free period for these patients ranged between 1 and 8 years (mean duration, 2.2 years). The number of VFs ranged from 1 to 9 , and five of the patients with VFs had also had a fragility non-vertebral fracture (Colles' fracture $(n=2)$, forearm fracture $(n=1)$ and hip fracture $(n=2))$.

\section{Sample processing}

Whole blood was collected in tubes containing clot activator and left at room temperature for at least $10 \mathrm{~min}$ and less than an hour before centrifugation for $10 \mathrm{~min}$ at $1900 \mathrm{~g}$ and $4^{\circ} \mathrm{C}$. Serum phase was transferred to conical tubes and centrifuged again for $10 \mathrm{~min}$ at $16000 \mathrm{~g}$ and $4^{\circ} \mathrm{C}$ in a fixed-angle rotor to remove additional cellular nucleic acids attached to cell debris. Serum was frozen in aliquots at $-80^{\circ} \mathrm{C}$ until further processing. 
To process frozen lysates, samples were incubated at $37^{\circ} \mathrm{C}$ in a water bath until completely thawed and salts were dissolved. After thawing, serum samples were centrifuged for $5 \mathrm{~min}$ at $16000 \boldsymbol{g}$ and $4^{\circ} \mathrm{C}$ to remove cryoprecipitates.

Levels of total alkaline phosphatase (ALP), total calcium, albumin and creatinine were determined by routine laboratory methods.

\section{Selection of microRNAs primer assays}

Selection of microRNAs was based on existing literature on specific microRNAs that were reported to correlate with bone metabolism both in serum and tissue samples. In addition, the following databases 1. miRBase (14), 2. DIANA TOOLS (15), 3. PicTar (16), 4. miRDB (17), 5. TargetScanHuman (18), 6. miRGator (19) and 7. microRNA (20) were searched to identify biological targets of microRNAs in humans, searching for $8 \mathrm{mer}, 7 \mathrm{mer}$ and 6 mer sites that match the seed region for each microRNA, using conserved sites and the best cumulative scores. Twelve microRNAs that fulfilled the above criteria were finally selected for analysis (Table 1). We additionally measured miR-422 and miR-133a that were previously reported in circulating monocytes of women with low $\operatorname{BMD}(21,22)$.

\section{Isolation of microRNAs from the serum}

miRNAs were extracted from $200 \mu \mathrm{L}$ of serum sample using the miRNeasy Serum/Plasma Kit, according to the manufacturer's instruction (Qiagen). During the purification process, a synthetic RNA sequence (spike ins: C. elegans miR-39) was added in appropriate amounts to serum preparations after homogenization with the QIAzol lysis reagent to control for variations in recovery

Table 1 Selected microRNAS and gene targets.

\begin{tabular}{|c|c|}
\hline hsa-miRNA & Gene \\
\hline \multirow[t]{4}{*}{ hsa-miR-21-5p } & SPRY1 \\
\hline & $D K K 2$ \\
\hline & $B M P 3$ \\
\hline & SMAD7 \\
\hline \multirow[t]{2}{*}{ hsa-miR-23a-3p } & RUNX2 \\
\hline & SATB2 \\
\hline \multirow[t]{2}{*}{ hsa-miR-24-2-5p } & $C A L B 1$ \\
\hline & SATB2 \\
\hline \multirow[t]{3}{*}{ hsa-miR-26a-5p } & COL19A1 \\
\hline & COL10A1 \\
\hline & EPHA5 \\
\hline \multirow[t]{4}{*}{ hsa-miR-29a } & COL3A1 \\
\hline & COL5A3 \\
\hline & PTHLH \\
\hline & DUSP2 \\
\hline \multirow[t]{3}{*}{ hsa-miR-33a-5p } & WIF1 \\
\hline & $D K K 2$ \\
\hline & OSTF1 \\
\hline \multirow[t]{3}{*}{ hsa-miR-124-3p } & NFATC1 \\
\hline & NFATC2 \\
\hline & HDAC5 \\
\hline \multirow[t]{4}{*}{ hsa-miR-135b-5p } & FOX01 \\
\hline & $A C V R 1 B$ \\
\hline & SMAD5 \\
\hline & SIRT1 \\
\hline \multirow[t]{3}{*}{ hsa-miR-214-3p } & $V D R$ \\
\hline & CTNNB1 \\
\hline & ATP2A3 \\
\hline \multirow[t]{4}{*}{ hsa-miR-218-5p } & SOST \\
\hline & SFRP2 \\
\hline & COL1A1 \\
\hline & EPHA5 \\
\hline \multirow[t]{2}{*}{ hsa-miR-335-3p } & $S P A R C^{*}$ \\
\hline & $D K K 1$ \\
\hline hsa-miR-2861 & HDAC5 \\
\hline
\end{tabular}

Gene function (from human miR database cross-refs with score $>90$ )

Sprouty homolog 1, antagonist of FGF signaling

Dickkopf Wnt signaling pathway inhibitor 2

Bone morphogenetic protein 3

SMAD family member 7

Runt-related transcription factor 2

SATB homeobox 2

Calbindin 1

SATB homeobox 2

Collagen, type $X I X$, alpha 1

Collagen, type $X$, alpha 1

$E P H$ receptor $A 5$

Collagen, type III, alpha 1

Collagen, type $V$, alpha 3

Parathyroid hormone-like hormone

Dual specificity phosphatase 2

Wnt inhibitory factor 1

Dickkopf Wnt signaling pathway inhibitor 2

Osteoclast stimulating factor 1

Nuclear factor of activated T-cells, cytoplasmic, 1

Nuclear factor of activated T-cells, cytoplasmic, 2

Histone deacetylase 5

Forkhead box 01

Activin A receptor, type IB

SMAD family member 5

Sirtuin 1

Vitamin $D(1,25$-dihydroxyvitamin $D 3)$ receptor

Catenin (cadherin-associated protein), beta 1

ATPase, Ca++ transporting, ubiquitous

Sclerostin

Frizzled-related protein 2

Collagen, type I, alpha 1

EPH receptor $A 5$

Secreted protein, acidic, cysteine-rich (osteonectin)

Dickkopf Wnt signaling pathway inhibitor 1

Histone deacetylase 5 
Table 2 miScript primer assays.

\begin{tabular}{l}
\hline Gene symbol \\
\hline hsa-miR-21-5p \\
hsa-miR-23a-3p \\
hsa-miR-24-2-5p \\
hsa-miR-26a-5p \\
hsa-miR-29a-3p \\
hsa-miR-33a-5p \\
hsa-miR-124-3p \\
hsa-miR-135b-5p \\
hsa-miR-214-3p \\
hsa-miR-218-5p \\
hsa-miR-335-5p \\
hsa-miR-2861 \\
hsa-miR-133a-3p \\
hsa-miR-422a
\end{tabular}

\begin{tabular}{l}
\hline Description \\
\hline Mature microRNA \\
Mature microRNA \\
Mature microRNA \\
Mature microRNA \\
Mature microRNA \\
Mature microRNA \\
Mature microRNA \\
Mature microRNA \\
Mature microRNA \\
Mature microRNA \\
Mature microRNA \\
Mature microRNA \\
Mature microRNA \\
Mature microRNA
\end{tabular}

\begin{tabular}{l}
\hline Assay catalogue \# (Qiagen) \\
\hline MS00009079 \\
MS00031633 \\
MS00009205 \\
MS00029239 \\
MS00003262 \\
MS00003304 \\
MS00006622 \\
MS00003472 \\
MS00031605 \\
MS00006769 \\
MS00003976 \\
MS00031423 \\
MS00004172
\end{tabular}

\begin{tabular}{l}
\hline Sequence \\
\hline 5'UAGCUUAUCAGACUGAUGUUGA \\
5'AUCACAUUGCCAGGGAUUUCC \\
5'UGCCUACUGAGCUGAAACACAG \\
5'UUCAAGUAAUCCAGGAUAGGCU \\
5'UAGCACCAUCUGAAAUCGGUUA \\
5'GUGCAUUGUAGUUGCAUUGCA \\
5'UAAGGCACGCGGUGAAUGCC \\
5'UAUGGCUUUUCAUUCCUAUGUGA \\
5'ACAGCAGGCACAGACAGGCAGU \\
5'UUGUGCUUGAUCUAACCAUGU \\
5'UCAAGAGCAAUAACGAAAAAUGU \\
5'GGGGCCUGGCGGUGGGCGG \\
5'UUUGGUCCCCUUCAACCAGCUG \\
5'ACUGGACUUAGGGUCAGAAGGC
\end{tabular}

and amplification efficiency between RNA preparations. $1.25 \mathrm{mg} / \mathrm{mL}$ bacteriophage MS2 RNA was used as a carrier. Total RNA was eluted with $20 \mu \mathrm{L}$ of RNAse-free water.

\section{Reverse transcription and PCR analysis}

After elution, $2 \mu \mathrm{L}$ were used as a template for reverse transcription with the miScript II RT Kit (Qiagen). After completion, each reverse transcription reaction was diluted to $220 \mu \mathrm{L}$ with RNase-free water, and $1 \mu \mathrm{L}$ was used as template for each single miR assay (Table 2), according to the manufacturer's instruction (Qiagen). A panel of 3 invariant miRs, two snoRNAs (SNORD95 and SNORD96A) and one snRNA (RNU6-2) was used to normalize for variability in sample loading and real-time RT-PCR efficiency.

Cycling was performed under standardized conditions with 2× QuantiTect SYBR Green PCR Master Mix on the QIAGEN Rotor-Gene Q (Corbett Rotor-Gene 6000) real-time PCR cycler. Performance of PCR was done in triplicates.

\section{Statistical analysis}

Resultant data on mean CT values for each microRNA were exported and uploaded to the QIAGEN Website for analysis, where a classic $\Delta \Delta C_{\mathrm{T}}$ calculation and a $\log 2$ transformation provided normalized fold-difference values for the microRNA targets. The software used for analysis was supported by SABiosciences (miR primer assay data analysis version 3.5, GeneGlobe Data Analysis). Mean $C_{\mathrm{T}}$ values less than 33 were used as the cutoff threshold as recommended by the software instructions. All analyses were based on fold-change ( $\left.2^{\text {(-Delta Delta Ct) }}\right)$ defined as the normalized gene expression $\left(2^{(- \text {Delta }} \mathrm{Ct}\right)$ in the patient's sample (postmenopausal osteoporotic women with or without fractures) divided by the normalized gene expression $\left(2^{(- \text {Delta } \mathrm{Ct})}\right)$ in the control sample (healthy controls). Fold-change values less than one are indicative of downregulation of the gene expression, and foldchange values greater than one indicate upregulation, accordingly. The $P$ values were calculated based on a Student's $t$-test of the replicate $2^{\text {(-Delta Ct) }}$ values for each gene in the control group (healthy postmenopausal women) and osteoporotic women.

Data on biochemical and anthropometric results are given as means \pm standard deviation (s.D.). Normal distribution was evaluated by the Kolmogorov-Smirnov test. One-way ANOVA followed by Bonferroni's multiple comparison tests and/or Dunnett test or a Kruskal-Wallis test was performed to assess the differences between groups, as applicable. Pearson correlation coefficient or Spearman's rank correlation coefficient was used for associations between relative serum expression of miRs and bone parameters of the study population, as applicable. Receiver-operating characteristic curves were analyzed to assess the specificity and sensitivity of specific microRNAs for osteoporotic postmenopausal women with VFs. All $P$ values are two-sided and a value of $P<0.05$ was considered as statistically significant.

\section{Results}

\section{Quality control}

To determine the efficiency of RNA extractions and/or the presence of inhibitors in cDNA synthesis or in PCR, we quantified the levels of the spike-in added before RNA extraction by real-time PCR. All cDNAs expressed the spike-in control at normal levels $\left(C_{\mathrm{t}}<22\right.$ cycles). Statistical 
Table 3 Anthropometric characteristics, biochemical values and $T$-scores of the study population (mean \pm s.D.).

\begin{tabular}{l}
\hline Parameters \\
\hline Age (years) \\
BMI $\left(\mathrm{kg} / \mathrm{m}^{2}\right)$ \\
Age at menopause \\
Serum calcium levels $(\mathrm{mg} / \mathrm{dL})$ \\
Serum creatinine levels $(\mathrm{mg} / \mathrm{dL})$ \\
Serum ALP levels (U/L) \\
$T$-Score TH \\
$T$-Score FN \\
$T$-Score LS
\end{tabular}

\begin{tabular}{c}
\hline Controls $(n=30)$ \\
\hline $68 \pm 5$ \\
$28 \pm 4$ \\
$52 \pm 2$ \\
$9.5 \pm 0.2$ \\
$0.7 \pm 0.1$ \\
$57 \pm 16$ \\
$-0.90 \pm 0.4$ \\
$-0.93 \pm 0.3$ \\
$-0.15 \pm 1$
\end{tabular}

\begin{tabular}{c}
\hline Group $1(n=35)$ \\
\hline $68 \pm 7$ \\
$27 \pm 5$ \\
$49 \pm 5$ \\
$9.4 \pm 0.4$ \\
$0.7 \pm 0.1$ \\
$73.4 \pm 20 *$ \\
$-2.19 \pm 1.02 *$ \\
$-2.53 \pm 0.80^{*}$ \\
$-2.67 \pm 0.67 *$
\end{tabular}

\begin{tabular}{c}
\hline Group $2(n=35)$ \\
\hline $71 \pm 7$ \\
$28 \pm 5$ \\
$48 \pm 5$ \\
$9.5 \pm 0.5$ \\
$0.8 \pm 0.2$ \\
$85.6 \pm 19.4^{*}$ \\
$-2.540 \pm 0.75^{*}$ \\
$-2.65 \pm 0.57^{*}$ \\
$-2.81 \pm 1.05^{*}$
\end{tabular}

Group 1: Postmenopausal women with low bone mass without vertebral fractures. Group 2: Postmenopausal women with low bone mass and vertebral fractures.

${ }^{*} P$ value $<0.05$ when comparisons were made with the control group. ${ }^{*} P$ value $<0.05$ when comparisons were made between group 1 and group 2 . BMI, body mass index; FN, femoral neck; LS, lumbar spine; TH, total hip.

analysis confirmed the absence of outliers in the $C_{\mathrm{t}}$ values for the spike-in.

\section{Study population}

Anthropometric characteristics, biochemical parameters and BMD values of the study population are depicted in Table 3. Bone mineral density did not differ significantly between patients with osteoporosis/osteopenia and VFs compared with patients without VFs.

\section{Differential expression of selected microRNAs in the serum of osteoporotic patients}

Two circulating miRs from the 14 tested (miR-124-3p and miR-2861) were significantly higher ( $>2$ fold-change), whereas 3 miRs (miR-21-5p, miR-23a-3p and miR-29a-3p) were significantly lower (fold-change $<1$ ) in the serum of osteoporotic patients $(n=70)$ compared with controls $(n=30)$ (Table 4).
Similar results were obtained when osteoporotic patients with (group 2) and without (group 1) VFs were compared with the controls separately (Fig. 1). In the subgroup comparisons between the two groups of patients with low bone mass, miR-21-5p was significantly lower in those with VF (fold-change; 0.70, CI; 0.48, 0.92, $P=0.04$ ).

No correlation was found between the relative expression of the tested miRs and age, creatinine levels, calcium levels, BMD or $T$-score at any skeletal site or the number of fractures.

A ROC analysis was performed for the differentially expressed microRNAs in the serum of osteoporotic/ osteopenic patients to assess their potential value in distinguishing between women with and without VFs. The associated area under the curve (AUC) of miR-21-5p was $0.66(95 \%$ CI $0.50,0.81, P=0.040)$ showing a sensitivity of $66 \%$ and $71 \%$ and a specificity of $71 \%$ (Fig. 2).

The AUC for miR-29a-3p was 0.61 (95\% CI 0.45-0.77, $P=0.180$ ) and for miR-23a-3p was 0.63 (95\% CI 0.47-0.79, $P=0.115)$.

Table 4 Fold-change of miRs expression in patients with low bone mass compared with controls.

\begin{tabular}{l}
\hline Genes \\
\hline hsa-miR-21-5p \\
hsa-miR-23a-3p \\
hsa-miR-24-2-5p \\
hsa-miR-26a-5p \\
hsa-miR-29a-3p \\
hsa-miR-33a-5p \\
hsa-miR-124-3p \\
hsa-miR-135b-5p \\
hsa-miR-214-3p \\
hsa-miR-218-5p \\
hsa-miR-335-5p \\
hsa-miR-2861 \\
hsa-miR-133a-3p \\
hsa-miR-422a
\end{tabular}

\begin{tabular}{c}
\hline Fold-change \\
\hline $\mathbf{0 . 6 0 9}$ \\
$\mathbf{0 . 4 6 4}$ \\
1.451 \\
1.507 \\
$\mathbf{0 . 4 8 9}$ \\
14.760 \\
$\mathbf{4 . 0 1 1}$ \\
2.756 \\
1.487 \\
2.034 \\
1.304 \\
$\mathbf{4 . 3 0 4}$ \\
1.1319 \\
0.9629
\end{tabular}

\begin{tabular}{ll}
\multicolumn{1}{c}{$95 \% \mathrm{CI}$} \\
\hline$(0.44,0.78)$ \\
$(0.25,0.68)$ \\
$(0.72,2.18)$ \\
$(0.01,3.00)$ \\
$(0.21,0.77)$ \\
$(0.00001,43.60)$ \\
$(2.04,6.20)$ \\
$(0.45,5.06)$ \\
$(0.72,2.25)$ \\
$(0.55,3.52)$ \\
$(0.68,1.93)$ \\
$(2.25,6.35)$ \\
$(0.93,1.34)$ \\
$(0.82,1.10)$
\end{tabular}

\begin{tabular}{r}
\hline $\boldsymbol{P}$ Value \\
\hline $\mathbf{0 . 0 4 0}$ \\
$<\mathbf{0 . 0 0 1}$ \\
0.187 \\
0.208 \\
$<\mathbf{0 . 0 0 1}$ \\
0.624 \\
$\mathbf{0 . 0 1 3}$ \\
0.473 \\
0.178 \\
0.608 \\
0.213 \\
$\mathbf{0 . 0 0 8}$ \\
0.510 \\
0.531
\end{tabular}




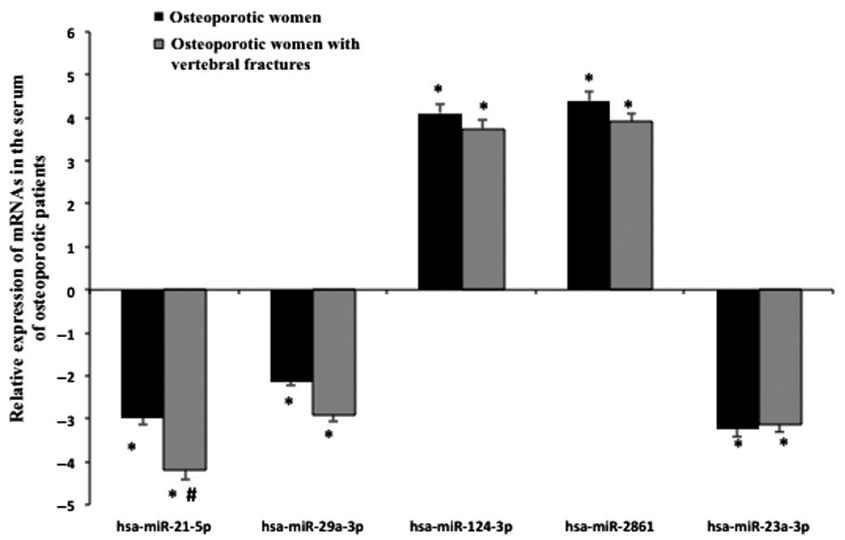

Figure 1

Relative expression of microRNAs in the serum of patients with low BMD and fractures compared with controls. * $P$ value $<0.05$ when comparisons are made with controls, " $P$ value $<0.05$ when comparisons are made between osteoporotic women with and without vertebral fractures.

\section{Discussion}

In this study, we investigated miR patterns in the serum of osteoporotic and osteopenic patients with and without VFs. Among the miRs that were differentially expressed in the serum of our patient population compared with controls, namely miR-124-3p, miR-2861, miR-21-5p, miR-23a-3p and miR-29a-3p, we found that miR-21-5p serum levels were significantly lower in patients who had sustained VFs compared with those without fractures.

Studies on circulating miRs in patients with bone diseases have only recently been reported, and some of them are restricted to circulating monocytes, as these are the cells that can differentiate into osteoclasts and secrete osteoclastogenic factors (21). In the early studies of Wang et al. (22) and Cao et al. (21), miR-133a and miR-422a respectively, were found to be significantly higher in circulating monocytes of women with low BMD compared with women with high BMD. In both studies, the upregulated miRNAs were negative regulators of osteoclastogenesis $(21,22)$, which is in line with the role of circulating monocytes in bone homeostasis as precursors of the osteoclasts. In our study, we found no difference in the serum levels of miR-133a and miR-422a between patients with low BMD with or without VFs and controls. Our results would appear to be in conflict with these early studies. However, as Wang et al. (22) reported in their study, miR-133a and miR-422a seem to display a selective, monocyte-specific role in postmenopausal osteoporosis, as in circulating B-cells, miR-133a expression was no longer upregulated. In addition, it is worth noting that, according to several databases $(14,15$, $16,17,18,19,20)$, these two miRs do not seem to play significant role in the RNA expression of osteoblast- or osteoclast-related genes in humans.

In our study, serum levels of miR-124-3p and miR2861 were higher in postmenopausal women with low bone mass compared with those with normal BMD. MiR124-3p has been reported to inhibit osteoclastogenesis by suppressing nuclear factor of activated T-cells (NFATc1) and receptor activator of nuclear factor kappa-B ligand (RANKL)-mediated osteoclast differentiation of mouse bone marrow macrophages (23). MiR-2861, on the other hand, targets histone deacetylase 5 (HDAC5), a transcriptional co-repressor that antagonizes Runx2-mediated bone formation (24). Therefore, the increased expression of miR2861 and miR-124-3p in the serum of our patients with low bone mass could reflect a compensatory mechanism of the bone tissue in response to menopause-induced bone loss, enhancing osteoblast differentiation (25) and inhibiting osteoclastogenesis respectively.

\section{$\operatorname{miR} 21-5 p$}

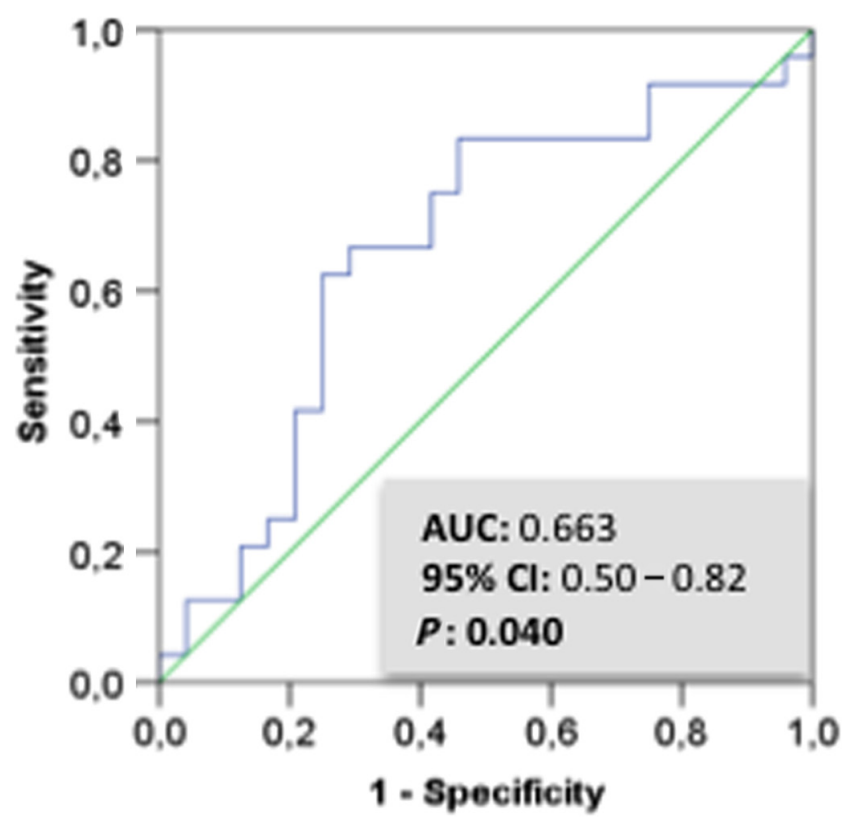

Figure 2

Area under the curve (AUC) of receiver-operating characteristic (ROC) for miR-21-5p and ROC curve. AUC, 95\% confidence interval $(95 \% \mathrm{Cl})$, and nominal $P$ values are demonstrated. The relative expression of miR-21-5p is log-transformed. A full colour version of this figure is available at http://dx.doi. org/10.1530/EJE-16-0583. 
We have also found significantly lower levels of miR-21-5p, miR-23a-3p and miR-29a-3p in the serum of osteoporotic/osteopenic women compared with controls. Interestingly, serum levels of miR-21-5p were significantly lower in patients who had suffered at least one VF compared with those without fractures.

Induction of miR-21 regulates the expression of the genes sprouty homolog 1 (SPRY), an antagonist of FGF signaling, and programmed cell death 4 (PDCD4), a neoplastic transformation inhibitor (25). Through the transcriptional repression of SPRY and PDCD4 genes, miR-21 regulates the activation of c-Jun-N-terminal kinase (JNK/C-Jun) and extracellular signal-regulated kinase (ERK/NF-kb) signaling. Thus, decreased expression of miR-21-5p inhibits ERK and JNK activation by increases of Pdcd4 and Spry1 protein levels respectively, decreasing osteoclastogenesis (26). MiR-23-3p, on the other hand, is a known repressor of Runx-2 transcription.

Our results would appear to be in conflict with Seeliger et al. (10) and Panach et al. (27) who demonstrated that miR-21-5p $(10,27)$ and miR-23a-3p (10) were upregulated in the serum and bone tissue of patients with osteoporotic fractures compared with controls. These discrepancies among studies are probably attributed to differences in the patients' populations and controls selected in each study. Seelinger et al. (10) compared the differential expression of circulating miRs in patients with osteoporotic hip fractures (men and women) vs patients with non-osteoporotic fractures, whereas Panach et al. included a group of osteoporotic patients with hip fractures compared with osteoarthritic patients with normal BMD values and no fractures (27). Fracture, however, in osteoporotic, osteoarthritic or normal bone tissue is a significant event that affects bone homeostasis and triggers significant changes in the molecular signaling pathways that interact during bone remodeling. In our study, controls were healthy postmenopausal women with normal BMD values who had never sustained a fracture before. Moreover, some changes in circulating miRs may be time dependent.

In our study, data on the specific time of fracture were not available, and this may have contributed to the different results that were obtained. In line with our results, Li et al. (28) also reported a significant decrease in miR-21-5p expression in cell-free plasma samples of osteoporotic Chinese women compared with women with osteopenia.

The miR-29 family is one of the best-characterized microRNA families with regard to osteoblast function (29), being key regulators of collagen expression, and they have not been described before in the serum of patients with osteoporosis. MicroRNA-29a additionally induces beta-catenin protein levels, indicating the activation of canonical Wnt signaling (30). MiR-29a, miR-29b and miR29c expression has been investigated in the serum and tissue of patients with osteosarcoma and was found to be significantly upregulated compared with normal controls (31). The serum levels of miR-29a and miR-29b were both independent prognostic factors of overall survival and disease-free survival of osteosarcoma patients. In our study, serum levels of miR-29a-3p were significantly lower in patients with low bone mass compared with controls, and in patients with VFs compared with those without VFs, albeit without reaching statistical significance.

Our study has certain limitations. The major limitation is its cross-sectional design, as the circulating miRs could be altered during the course of the disease. Furthermore, data regarding the specific time of fracture were not available. Another limitation is that a small fraction of our patients were not treatment naïve. However, the effect of the agents that they had received would be expected to be well washed out, when serum samples were obtained. Finally, our research was restricted to specific circulating miRs instead of performing an array analysis mainly due to funding restrains. However, the miRs that were finally selected are known as immediate gene regulators of bone metabolism and definitely conserved in humans.

Our data are compatible with the known pathophysiological mechanisms in bone biology, thereby supporting and suggesting the design of prospective studies to establish a critical role of miRs in the evaluation of bone strength attenuation.

In conclusion, we have found a differential expression of specific miRs such as miR-21-5p in the serum of osteoporotic/osteopenic patients with VFs compared with patients without VFs. Identification of miR signatures in the serum of patients with low BMD and fractures, which unlike classical markers, can reflect the cellular and molecular processes involved, is currently under intensive investigation and is considered a critical step toward the optimization of diagnosis and treatment of bone diseases.

Declaration of interest

The authors declare that there is no conflict of interest that could be perceived as prejudicing the impartiality of the research reported.

\section{Funding}

This research did not receive any specific grant from any funding agency in the public, commercial or not-for-profit sector. 
Acknowledgments

The authors thank A Michopoulos and M Tziomaki for technical assistance.

\section{References}

1 Raisz LG. Pathogenesis of osteoporosis: concepts, conflicts, and prospects. Journal of Clinical Investigation 2005115 3318-3325. (doi:10.1172/JCI27071)

2 Zaidi M. Skeletal remodeling in health and disease. Nature Medicine 200713 791-801. (doi:10.1038/nm1593)

3 Kanis JA, Oden A, Johnell O, Johansson H, De Laet C, Brown J, Burckhardt P, Cooper C, Christiansen C, Cummings $\mathrm{S}$ et al. The use of clinical risk factors enhances the performance of BMD in the prediction of hip and osteoporotic fractures in men and women. Osteoporosis International 200718 1033-1046. (doi:10.1007/s00198-007-0343-y)

4 Siris ES, Adler R, Bilezikian J, Bolognese M, Dawson-Hughes B, Favus MJ, Harris ST, Jan de Beur SM, Khosla S, Lane NE et al. The clinical diagnosis of osteoporosis: a position statement from the National Bone Health Alliance Working Group. Osteoporosis International 201425 1439-1443. (doi:10.1007/s00198-014-2655-z)

5 Schuit SC, van der Klift M, Weel AE, de Laet CE, Burger H, Seeman E, Hofman A, Uitterlinden AG, van Leeuwen JP \& Pols HA. Fracture incidence and association with bone mineral density in elderly men and women: the Rotterdam Study. Bone 200434 195-202. (doi:10.1016/j.bone.2003.10.001)

6 Silverman SL \& Calderon AD. The utility and limitations of FRAX: a US perspective. Current Osteoporosis Reports 20108 192-197. (doi:10.1007/s11914-010-0032-1)

7 Esteller M. Cancer epigenetics for the 21st century: what's next? Genes and Cancer 20112 604-606. (doi:10.1177/1947601911423096)

8 He L \& Hannon GJ. MicroRNAs: small RNAs with a big role in gene regulation. Nature Reviews Genetics 20045 522-531. (doi:10.1038/ $\operatorname{nrg} 1379)$

9 van Wijnen AJ, van de Peppel J, van Leeuwen JP, Lian JB, Stein GS, Westendorf JJ, Oursler MJ, Im HJ, Taipaleenmaki H, Hesse $\mathrm{E}$ et al. MicroRNA functions in osteogenesis and dysfunctions in osteoporosis. Current Osteoporosis Reports 201311 72-82. (doi:10.1007/s11914-013-0143-6)

10 Seeliger C, Karpinski K, Haug AT, Vester H, Schmitt A, Bauer JS \& van Griensven M. Five freely circulating miRNAs and bone tissue miRNAs are associated with osteoporotic fractures. Journal of Bone and Mineral Research 201429 1718-1728. (doi:10.1002/jbmr.2175)

11 Weilner S, Skalicky S, Salzer B, Keider V, Wagner M, Hildner F, Gabriel C, Dovjak P, Pietschmann P, Grillari-Voglauer R et al. Differentially circulating miRNAs after recent osteoporotic fractures can influence osteogenic differentiation. Bone 201579 43-51. (doi:10.1016/j.bone.2015.05.027)

12 Torricelli P, Fini M, Giavaresi G \& Giardino R. Human osteoblast cultures from osteoporotic and healthy bone: biochemical markers and cytokine expression in basal conditions and in response to 1,25(OH)2D3. Artificial Cells, Blood Substitutes, and Immobilization Biotechnology 200230 219-227. (doi:10.1081/BIO-120004341)

13 Liu YZ, Dvornyk V, Lu Y, Shen H, Lappe JM, Recker RR \& Deng HW. A novel pathophysiological mechanism for osteoporosis suggested by an in vivo gene expression study of circulating monocytes. Journal of Biological Chemistry 2005280 29011-29016. (doi:10.1074/jbc. M501164200)

14 Kozomara A \& Griffiths-Jones S. miRBase: annotating high confidence microRNAs using deep sequencing data. Nucleic Acids Research 2014 42 D68-D73. (doi:10.1093/nar/gkt1181)
15 Paraskevopoulou MD, Georgakilas G, Kostoulas N, Vlachos IS, Vergoulis T, Reczko M, Filippidis C, Dalamagas T \& Hatzigeorgiou AG. DIANA-microT web server v5.0: service integration into miRNA functional analysis workflows. Nucleic Acids Research 201341 W169-W173. (doi:10.1093/nar/gkt393)

16 Reczko M, Maragkakis M, Alexiou P, Grosse I \& Hatzigeorgiou AG. Functional microRNA targets in protein coding sequences. Bioinformatics 201228 771-776. (doi:10.1093/bioinformatics/bts043)

17 Wong N \& Wang X. miRDB: an online resource for microRNA target prediction and functional annotations. Nucleic Acids Research 201543 D146-D152. (doi:10.1093/nar/gku1104)

18 Agarwal V, Bell GW, Nam JW \& Bartel DP. Predicting effective microRNA target sites in mammalian mRNAs. Elife 20154 1-38. (doi:10.7554/elife.05005)

19 Cho S, Jang I, Jun Y, Yoon S, Ko M, Kwon Y, Choi I, Chang H, Ryu D, Lee B et al. MiRGator v3.0: a microRNA portal for deep sequencing, expression profiling and mRNA targeting. Nucleic Acids Research 2013 41 D252-D257. (doi:10.1093/nar/gks1168)

20 Betel D, Koppal A, Agius P, Sander C \& Leslie C. Comprehensive modeling of microRNA targets predicts functional non-conserved and non-canonical sites. Genome Biology 201011 R90. (doi:10.1186/ gb-2010-11-8-r90)

21 Cao Z, Moore BT, Wang Y, Peng XH, Lappe JM, Recker RR \& Xiao P. MiR-422a as a potential cellular microRNA biomarker for postmenopausal osteoporosis. PLoS ONE 20149 e97098. (doi:10.1371/ journal.pone.0097098)

22 Wang Y, Li L, Moore BT, Peng XH, Fang X, Lappe JM, Recker RR $\&$ Xiao P. MiR-133a in human circulating monocytes: a potential biomarker associated with postmenopausal osteoporosis. PLOS ONE 20127 e34641. (doi:10.1371/journal.pone.0034641)

23 Lee Y, Kim HJ, Park CK, Kim YG, Lee HJ, Kim JY \& Kim HH. MicroRNA-124 regulates osteoclast differentiation. Bone 201356 383-389. (doi:10.1016/j.bone.2013.07.007)

24 McGee-Lawrence ME \& Westendorf JJ. Histone deacetylases in skeletal development and bone mass maintenance. Gene 2011474 1-11. (doi:10.1016/j.gene.2010.12.003)

25 Hu R, Liu W, Li H, Yang L, Chen C, Xia ZY, Guo LJ, Xie H, Zhou HD, Wu XP et al. A Runx2/miR-3960/miR-2861 regulatory feedback loop during mouse osteoblast differentiation. Journal of Biological Chemistry 2011286 12328-12339. (doi:10.1074/jbc.M110.176099)

26 Shen L, Ling M, Li Y, Xu Y, Zhou Y, Ye J, Pang Y, Zhao Y, Jiang R, Zhang J et al. Feedback regulations of miR-21 and MAPKs via Pdcd4 and Spry1 are involved in arsenite-induced cell malignant transformation. PLOS ONE 20138 e57652. (doi:10.1371/journal.pone.0057652)

27 Panach L, Mifsut D, Tarin JJ, Cano A \& Garcia-Perez MA. Serum circulating microRNAs as biomarkers of osteoporotic fracture. Calcified Tissue International 201597 495-505. (doi:10.1007/s00223-0150036-z)

28 Li H, Wang Z, Fu Q \& Zhang J. Plasma miRNA levels correlate with sensitivity to bone mineral density in postmenopausal osteoporosis patients. Biomarkers 201419 553-556. (doi:10.3109/13547 50X.2014.935957)

29 Kapinas K \& Delany AM. MicroRNA biogenesis and regulation of bone remodeling. Arthritis Research and Therapy 201113 220. (doi:10.1186/ ar3325)

30 Kapinas K, Kessler C, Ricks T, Gronowicz G \& Delany AM. miR-29 modulates Wnt signaling in human osteoblasts through a positive feedback loop. Journal of Biological Chemistry 2010285 25221-25231. (doi:10.1074/jbc.M110.116137)

31 Hong Q, Fang J, Pang Y \& Zheng J. Prognostic value of the microRNA-29 family in patients with primary osteosarcomas. Medical Oncology 201431 37. (doi:10.1007/s12032-014-0037-1)

Received 10 July 2016

Revised version received 18 October 2016

Accepted 11 November 2016 